\title{
SYNCHROTRON RADIATION and CATALYTIC SCIENCE
}

Christopher Hardacre, (University of Manchester); Andrew M. Beale, (University College London, Research Complex at Harwell); Emma K. Gibson (University of Glasgow); Josephine B. M Goodall (Cardiff University, Research Complex at Harwell); Alex Goguet (Queens University Belfast); Simon A. Kondrat (Loughborough University); Grazia Malta (Cardiff University); Cristina Stere (University of Manchester); Peter P. Wells University of Southampton, Diamond Light Source) Graham J. Hutchings (Cardiff University); C. Richard A. Catlow (University of Cardiff, University College London

\section{Introduction}

Techniques employing synchrotron radiation (SR) have had a major and growing impact on catalytic science. They have made key contributions to our understanding of structural properties of catalytic systems and of structural changes during the operation of a catalytic process. They can also improve our understanding of electronic and vibrational properties which can contribute to the understanding of mechanisms. SR techniques are now key components of the experimental tool box of the catalytic scientist.

The most widely used range of techniques in catalysis studies is possibly $X$-Ray Absorption Fine Structure (XAFS) comprising both the near edge (XANES) and Extended (EXAFS) regions of the X-Ray absorption spectrum. Catalytic science benefits from the element specific data that these techniques provide on oxidation state, coordination number and detailed local structure. As such they provide unique information on active site structures and structural changes.

$X$-Ray Powder Diffraction (XRPD), which with SR yields high quality data which can be refined using profile refinement (Rietveld) techniques, enables the resolution of the crystal structures of complex catalytic materials, such as microporous catalysts, which are only available as powders, while the intensity of SR enables single crystal studies on microcrystals; and since the 1990s it has also been possible to combine XAFS and XRPD allowing local and long-range structure to be obtained simultaneously. Moreover, tomographic techniques based on both spectroscopic and diffraction data allow the development of detailed 3D structures of heterogeneous catalytic particles. Catalytic science also benefits from a range of surface sensitive techniques including $X P S$ and surface diffraction; while recently SR based IR measurements are proving to be of growing value.

Catalytic science has profited hugely from the ability to undertake SR based experiments in situ, (while the catalyst is functioning) and in operando (as the product distribution is also monitored). The intensity of SR has also allowed increasingly fine time and spatial resolution, so that it is now possible, for example, to undertake kinetic in situ structural studies, spatially resolved along a catalytic reactor. SR data may also be collected in combination with other experimental techniques, where combined $X A F S / D R I F T S$ have proved to be particularly powerful. 
Developments in sources, instrumentation and data analysis techniques continue to extend the range and power of SR techniques in catalytic science. In this article, their growing impact is illustrated using a series of case studies based on work within the UK Catalysis Hub - a UK network of catalytic scientists, which has made extensive contributions to both the development and application of SR techniques in catalysis, especially using the facilities of the Diamond Light Source based on the UK Harwell Science and Innovation Campus.

\section{Development of multimodal tomography for microscale chemical imaging of catalysts under operational conditions}

By accident or by design, catalysts often possess a micro- and nano-structure which can influence performance, but which is rarely considered when correlating structure with function during in situ and/or operando studies. In recent years we have developed chemical imaging approaches to understand better the importance of the distribution of this multi-scale structures and how they evolve [1]. For this purpose, at Diamond Light Source, beamline I18, we have developed a multimodal tomography approach that allows for simultaneous collection of X-ray fluorescence, diffraction and absorption measurements on a functional material under operando conditions. In an exemplar study we characterised a packed bed micro-reactors $(500 \mu \mathrm{m}$ reactor containing $\mathrm{Co} / \mathrm{SiO}_{2}$ Fischer Tropsch synthesis (FTS) catalysts), enabling identification of the active species and correlation with performance $[2,3]$

In Figure 1 we show the multimodal data acquired in one shot of the X-ray beam. The data (particularly the XRD) are of sufficiently high quality to extract detailed structural insight; for example fitting of the XRD-CT patterns revealed no hexagonal close packed (HCP) cobalt but a mixture of cubic close packed (CCP) and intergrown Co (i.e. a random mixture of $\mathrm{CCP}$ and $\mathrm{HCP}$ layers). This degree of intergrowth can be directly correlated with the Ti modification on the silica support. When Ti is deposited before the $\mathrm{Co}$, it crystallises on the support as $\mathrm{TiO}_{2}$ anatase, increasing the strength of the metal support interaction and resulting in smaller, strained Co nanoparticles (a result of a higher degree of intergrowth) that are prone to reoxidation under FTS conditions (Figure 1). The spatially resolved nature of the measurements reveals that this reoxidation occurs only in regions with a high concentration of this $\mathrm{TiO}_{2}$.

When Ti is deposited after Co, anatase does not form, and the Co does not oxidise during FTS. In both cases, however, the degree of intergrowth (i.e. disorder) in the Co is linked to the concentration of $\mathrm{Ti}$ and not its crystallinity. The Co nanoparticles formed when $\mathrm{Ti}$ is deposited first are smaller and more active, yielding higher $\mathrm{CO}$ conversion, whereas when $\mathrm{Ti}$ is deposited second, the Co nanoparticles formed are larger and have greater selectivity towards the more desired longer-chain hydrocarbons.

The principal advantage of these tomographical approaches is their ability to provide information that resolves, spatially, signals concerning the microstructure of the sample that would otherwise be lost in a bulk measurement. Such local signals are simpler to interpret, since they are likely to contain fewer phases. Furthermore, studying intact materials rather than idealised powders allows for behaviour under industrially relevant 
conditions to be observed, mitigating some of the risk when trying to translate results from the lab to the plant. In addition, the background signal from the apparatus / cell can be readily separated. Recent advances in data processing allow for this information to be visualized in near real-time during experiments, allowing for informed decisions on its progress, enabling treatments and measurements to be adapted accordingly [4].

\section{The UK Catalysis Hub Block Allocation Group (BAG) Access to Diamond Light Source}

Within the catalysis community, access to synchrotron radiation techniques has primarily been the preserve of the experienced user groups and their academic offspring. However, this model fails in fulfilling the potential of the methodology and scientific advances that these groups have pioneered for several decades. The impact of this work is more keenly realised when it is exposed to an increased breadth of scientific challenges. In 2011, a fledgling catalysis consortium within the UK was formed, which in 2013 became the UK Catalysis Hub. Having a research base adjacent to Diamond Light Source, there was a determination to make better use of the central facilities. Discussions with Diamond led to the idea of a Block Allocation Group (BAG) for the new catalysis consortium on the core X-ray absorption beamline, B18. There was, however, a key difference in how this BAG was to be administered. The BAG access had an internal panel that prioritised access not only on the excellence of the underpinning science but also on bringing in new scientific challenges and user groups. Furthermore, the administrators of this access route - Peter Wells and Emma Gibson endeavoured to see the potential of the scientific proposals and collaborated with user groups to design the most appropriate experiments for the scientific challenge at hand.

This approach has proved to be hugely successful and makes a significant contribution to the publication output of the beamline - beyond what would be expected based on the number of assigned days. A couple of highlights of this approach are detailed below:

\section{An Artificial Rhodium Hydroformylase for Linear Aldehydes [5],}

As part of this initiative, there was a concerted effort to broaden the uptake of synchrotron radiation methods beyond heterogeneous catalysis. This project harnessed the catalytic properties of transition metal complexes - in this case $\mathrm{Rh}$ - and the specificity afforded through the development of artificial metalloenzymes (ArMs) for the selective hydroformylation of long-chain alkenes. The design approach utilises phosphine bioconjugation methods for anchoring of Rh complexes to protein scaffolds. The ArMs developed through this method had demonstrated an activity $10^{3}$ times higher than the conventional homogeneous Rh complex analogue. The unanswered question was the nature of the Rh centre in the ArM and how it was interacting with the protein scaffold. The XAFS data suggested that the initial $\mathrm{Rh}$ complex, $\mathrm{Rh}(\mathrm{acac})(\mathrm{CO})_{2}$, underwent loss of both $\mathrm{CO}$ ligands during the immobilisation and was bound to two $\mathrm{P}$ environments - as part of the phosphine bioconjugation. However, these phosphine groups are particularly large and there would be significant steric hinderance 
with two bound to the Rh centre. It was, therefore, postulated that, rather than $\mathrm{P}$, there could be a bound $\mathrm{S}$, from methionine, from the protein scaffold. To test this hypothesis, selenomethionine analogues were prepared, allowing for clear differentiation between Se and $\mathrm{P}$ coordination. There was a profound change in the XAFS between both samples, which were found to be consistent with Se coordination to the Rh centre. This provided a unique insight in that the complex was not solely binding to the protein through the introduced phospine linkages, but to other functionalities on the protein scaffold itself. This structural level of understanding of the ArMs would not have been possible without both the UK Catalysis Hub network and the steering of the BAG administration.

The behaviour of diphosphine ligands in iron-catalysed Negishi cross-coupling [6], In promoting the uptake of XAFS amongst the UK homogeneous catalysis community, a collaboration with Robin Bedford's group was initiated on the use of Earth-abundant Fe complexes for cross-coupling reactions - a potential alternative to scarce and expensive Pd based catalysts. To provide information on the active catalytic species, as opposed to the end 'resting state' complex, an operando continuous flow reactor was developed. In this approach, each spatial position becomes a static time point that allows the catalyst to be assessed under realistic concentrations and with a temporal resolution (determined by the length of the flow reactor) consistent with the whole breadth of the reaction profile. This study provided time-resolved changes in catalyst speciation that were entirely consistent with the reaction profile (measured both during the XAFS data acquisition and offline). The thrust of the work had been to build a library of diphosphine ligands that are crucial to catalytic activity. However, the XAFS investigations provided a very surprising insight - during catalysis the diphosphine ligand does not reside on the Fe centre. Instead, under reaction conditions the diphosphine ligand resides on the $\mathrm{Zn}$ co-catalyst (confirmed subsequently with ${ }^{31} \mathrm{P}$ NMR). Indeed, the XAFS confirmed that the homogeneous Fe complexes formed Fe nanoparticles soon after the start of reaction, which subsequently formed a bromoferrate as the deactivation product. The exact nature of the active iron centre is the subject of further investigations; however, it is proposed that the key role of the diphosphine ligand is to facilitate transmetallation between the Fe and $\mathrm{Zn}$ centres, which is an important step in understanding how Fe catalysts work for cross-coupling reactions and their future uptake as replacements for precious metals.

\section{Single-site gold catalysts for the acetylene hydrochlorination reaction}

A collaboration between several universities, industrial partners at Johnson Matthey, ISIS neutron source and Diamond Light Source, through the UK Catalysis Hub, has resulted in significant advances in the understanding of industrially relevant gold catalysts used in the production of vinyl chloride monomer (VCM). VCM is the key material for the manufacture of polyvinyl chloride (PVC), with over 40 million tons produced annually. The project has been highly successful with publications in Science [7] and ACS Catalysis [8] to date. Further, the findings were assisted through combined computational theoretical calculations enabled by the Catalysis Hub. 
The main route for the VCM production in China is the hydrochlorination of acetylene, traditionally catalysed by carbon-supported mercuric chloride. In 2013, the "Minamata convention on mercury", prohibited the use of mercury in VCM plants [9]. Following the prediction of Hutchings [10] that Au would be an effective catalyst for the VCM production, Johnson Matthey has recently validated an $\mathrm{Au} / \mathrm{C}$ catalyst for this largescale industrial process [11].

However, a full understanding of the nature of the gold during reaction has hindered catalyst design. Our study [7], reported the first detailed X-ray absorption spectroscopy (XAS) experiment, at B18 Diamond Light Source, which followed the behaviour of these catalysts during the reaction. Analysis of the Au L $\mathrm{L}_{3}$-edge XANES and EXAFS showed that the operating catalyst was comprised of atomically dispersed cationic gold in both $\mathrm{Au}(\mathrm{III})$ and $\mathrm{Au}(\mathrm{I})$ oxidation states (Figure 2a,b); showing analogies with the single-site homogeneous catalysis.

Follow up work, published in ACS Catalysis [8], investigated the role of reactants in the deactivation and the reaction mechanism of these $\mathrm{Au} / \mathrm{C}$ catalysts (Figure $2 \mathrm{c}$ ), via in situ XAS sequential gas flow experiments. Alternating between a reaction mixture of $\mathrm{HCl} / \mathrm{C}_{2} \mathrm{H}_{2}$ and the single reactant it was demonstrated that oxidative addition of $\mathrm{HCl}$ across an $\mathrm{Au}(\mathrm{I})$ chloride species requires concerted addition with $\mathrm{C}_{2} \mathrm{H}_{2}$, in accordance with the XAS measurements and the reaction kinetics. Exposure of the $\mathrm{Au} / \mathrm{C}$ catalyst to only $\mathrm{C}_{2} \mathrm{H}_{2}$ changed $\mathrm{Au}$ speciation and resulted in the formation of oligomeric acetylene species, as detected by inelastic neutron scattering (INS).

\section{Into the effect of NPT on low temperature methane oxidation over a $\mathrm{Pd} / \mathrm{Al}_{2} \mathrm{O}_{3}$ catalyst}

Stringent incoming regulations on methane emissions are of major concern for the automotive sector. One of the investigated solutions is the coupling of supported metal catalysts for methane oxidation with non-thermal plasma (NTP) to address the issue of low-temperature methane slip. Palladium-based catalysts are highly active for the oxidation of $\mathrm{CH}_{4}$ to $\mathrm{CO}_{2}$ and $\mathrm{H}_{2} \mathrm{O}$ but temperatures above $300^{\circ} \mathrm{C}$ are required which prevent them being effective under cold start conditions and operation with methane as the fuel. In contrast, high $\mathrm{CH}_{4}$ conversions with high selectivity to $\mathrm{CO}_{2}$ can be achieved when using a combined plasma-catalytic system. An in-depth understanding of what is happening in the plasma-catalyst system during real time and realistic reaction conditions would offer valuable information both to the industrial sector and academia, as very few in situ studies are available to date for any plasma-catalytic systems. For the first time, a XAS investigation of a hybrid plasma-catalyst system was carried out by a consortium of researchers using the B18 beamline at Diamond Light Source (Figure 3).

The state-of-the-art technique was developed to facilitate the investigation of the gasphase reaction environment during plasma operation, whilst simultaneously allowing the recording of XAFS spectra providing crucial insights into the impact of the plasma 
on the catalyst structure.[12] The study indicated a selective change in temperature for the Pd nanoparticles compared to the bulk of the catalyst and no significant structural changes of the catalyst. Therefore, the high activity was not due to a modification of the catalyst by the plasma under the reaction conditions investigated. Furthermore, the reported change in temperature was not sufficient to account for the observed methane conversion; therefore, the hypothesis that the role of the plasma for such system was merely to provide heating to the catalyst could be ruled out. The most reasonable explanation for the high $\mathrm{CH}_{4}$ conversions was attributed to the cleavage of the first $\mathrm{C}$ $\mathrm{H}$ bond in the gas phase, via electron impact reactions. Since this step is reported to be the rate-determining step for the methane oxidation reaction over the $\mathrm{Pd} / \mathrm{Al}_{2} \mathrm{O}_{3}$ catalyst, the NTP can be regarded as a 'positive perturbation' of the catalytic system [13], that opens up alternative reaction pathways, through a lower activation barrier than in the thermal catalytic systems.

This study demonstrated the value of combining the activity data with information on the catalyst structure and oxidation state during plasma operation in developing an understanding of the plasma effect on the catalyst and reaction. It highlighted the importance of in situ studies in bridging the knowledge gap for plasma-catalytic systems.

\section{Conclusions}

We hope this article has illustrated the range and power of synchrotron based techniques in gaining detailed information at the molecular level of structure and processes in catalytic systems. New sources will open up new opportunities. In particular, the advent of X-Ray Free Electron Laser (XFEL) will offer exciting challenges and opportunities for catalytic science by giving direct information on mechanisms.

\section{Acknowledgements}

UK Catalysis Hub is kindly thanked for resources and support provided via our membership of the UK Catalysis Hub Consortium and funded by EPSRC grant: EP/R026939/1, EP/R026815/1, EP/R026645/1, EP/R027129/1 or EP/M013219/1(biocatalysis))" 2013-2018 and EP/K014706/2, EP/K014668/1, $\mathrm{EP} / \mathrm{K} 014854 / 1, \mathrm{EP} / \mathrm{K} 014714 / 1$ or EP/M013219/1 - 2018 - present

The authors thank the European Synchrotron Radiation Facility and the Diamond Light Source for beam time (allocation numbers SP12986-1 and SP10306-9) (allocation numbers SP10306, SP11398, and SP15214 SP10306, and SP12601, SP10242, NT12064, NT12499, and NT14440)

\section{References}

1. F. Meirer and B. M. Weckhuysen, Nature Reviews Materials, 3, 324-340 (2018). https://doi.org/10.1038/s41578-018-0044-5 
2. S. W. T. Price, D. J. Martin, A. D. Parsons, W. A. Slawinski, A. Vamvakeros, S. J. Keylock, A. M. Beale and J. F. W. Mosselmans, Science Advances, 3(3), 10 (2017). doi: $\underline{10.1126 / \text { sciadv. } 1602838}$

3. A. M. Beale, S. D. M. Jacques, M. Di Michiel, J. F. W. Mosselmans, S. W. T. Price, P. Senecal, A. Vamvakeros and J. Paterson, Philosophical Transactions of the Royal Society a-Mathematical Physical and Engineering Sciences, 376(2110), (2018). https://doi.org/10.1098/rsta.2017.0057

4. A. D. Parsons, S. W. T. Price, N. Wadeson, M. Basham, A. M. Beale, A. W. Ashton, J. F. W. Mosselmans and P. D. Quinn, Journal of Synchrotron Radiation, 24 (1), 248256 (2018). https://doi.org/10.1107/S1600577516017756

5. A. Jarvis, L. Obrecht, P.J. Deuss, W. Laan, E.K. Gibson, P.P. Wells, P. Kamer, Angewandte Chemie 56(44), 13596 -13600 (2017). . doi: 10.1002/anie.201705753

6. A. Messinis S.L. Luckham, P.P Wells, D. Gianolio, E.K. Gibson, H.M. O'Brien, H.A. Sparkes, S.A. Davis J. Callison, D. Elorriaga, O. Hernandes-Faardo, R. Bedford, , Nature Catalysis 2 123-133 (2019) doi:10.1038/s41929-018-0197-Z

7. G. Malta, S. A. Kondrat, S. J. Freakley, C. J. Davies, L. Lu, S. Dawson, A. Thetford, E.K. Gibson, D. J. Morgan, W. Jones, P.P Wells, P. Johnston, C.R.A Catlow, C.J. Kiely, G.J. Hutchings Scienc, 355 (6332), 1399- 1403 (2017). DOI: 10.1126/science.aal3439

8.G. Malta, S. A. Kondrat, S. J. Freakley, C. J. Davies, S. Dawson, X. Liu, L. Lu, K. Dymkowski, F. Fernandez-Alonso, S. Mukhopadhyay, E. K. Gibson, P. P. Wells, S. F. Parker, C. J. Kiely, G. J. Hutchings, ACS Catalysis, 8(9), 8493-8505 (2018). https://doi.org/10.1021/acscatal.8b02232

9. http://www.mercuryconvention.org/

10. G. J. Hutchings,. Journal of Catalysis 96(1) 292- 295 (1985). DOI: 10.1016/00219517(85)90383-5

11. P. Johnston, N. Carthey, G.J. Hutchings. Journal of the American Chemical Society., 137(46), 14548- 14557 (2015). DOI: 10.1021/jacs.5b07752

12. E.K. Gibson. C.E. Stere, B. Curran-McAteer, W. Jones, G. Cibin, D. Gianolio, P.P Wells C.R.A.C Catlow. P. Collier, P. Hinde,C. Hardacre, Angewandte Chemie International Edition 56(32), $9351-9355 \quad$ (2017). https://doi.org/10.1002/anie.201703550

13. J.C. Whitehead, Frontiers of Chemical Science and Engineering 13(2), 264-273 (2019).

https://doi.org/10.1007/s11705-019-1794-3 


\section{Acknowledgments}

UK Catalysis Hub is kindly thanked for resources and support provided via our membership of the UK Catalysis Hub Consortium and funded by EPSRC grant: EP/R026939/1, EP/R026815/1, EP/R026645/1, EP/R027129/1 or EP/M013219/1(biocatalysis))" 2013-2018 and EP/K014706/2, EP/K014668/1, $\mathrm{EP} / \mathrm{K} 014854 / 1, \mathrm{EP} / \mathrm{K} 014714 / 1$ or EP/M013219/1 - 2018 - present

The authors thank the Diamond Light Source for beam time (allocation numbers SP12986-1 and SP10306-9) (allocation numbers SP10306, SP11398, and SP15214 SP10306, and SP12601, SP10242, NT12064, NT12499, and NT14440)

\section{Figures}

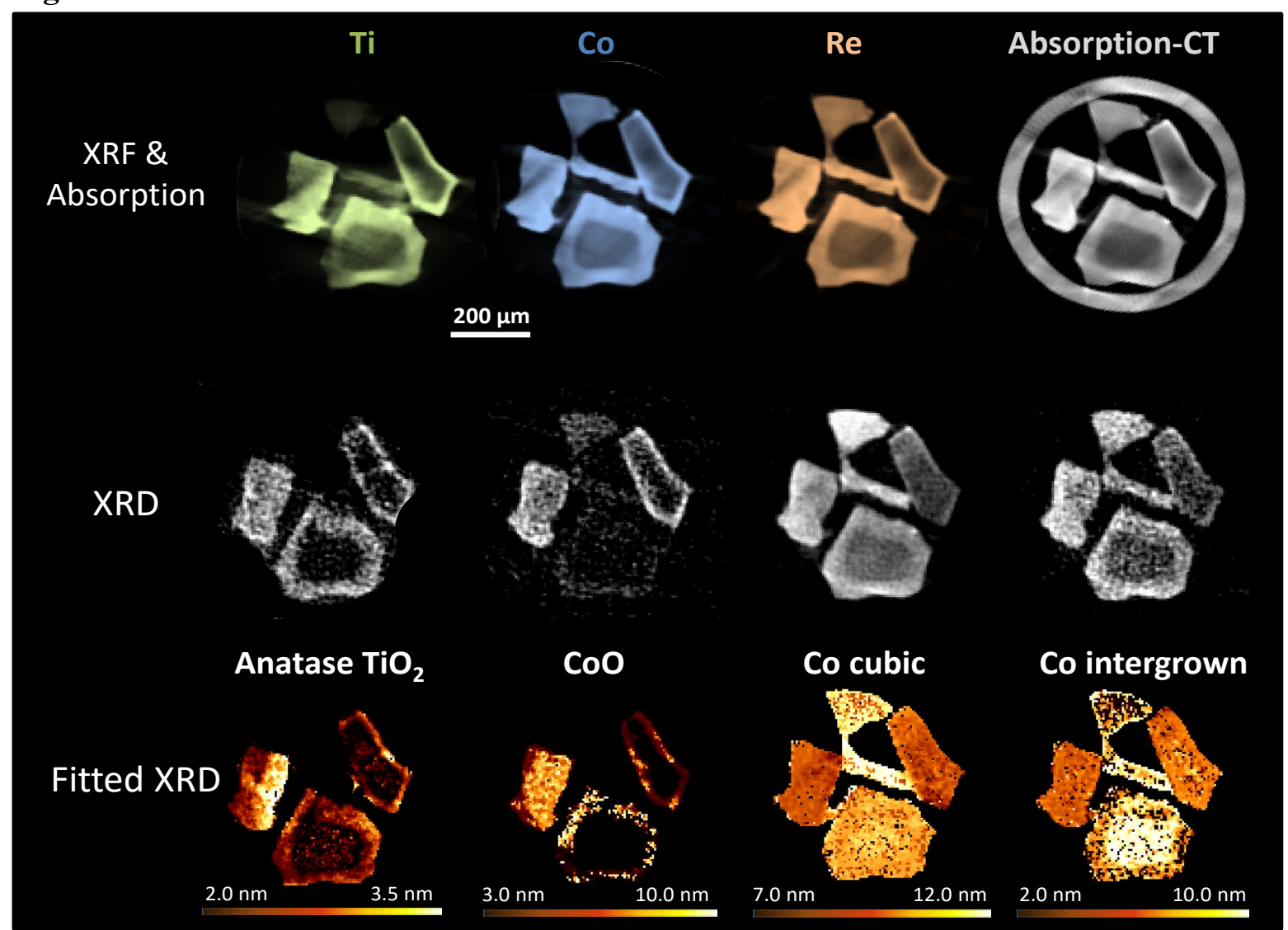

Figure 1. $\mathrm{Co} / \mathrm{SiO}_{2}$ catalyst structure during FTS. (Top) XRF-CT reconstructions showing elemental distributions during FTS at 2-bar pressure. Green, Ti; blue, Co; orange, Re. Absorption-CT reconstruction (grey) also shows the capillary wall surrounding the particles. (Middle) XRD-CT reconstructions of the catalyst revealing the phases present. (Bottom) Average crystallite size per pixel for each phase identified. Each pixel is $5 \mu \mathrm{m} \times 5 \mu \mathrm{m}$. 

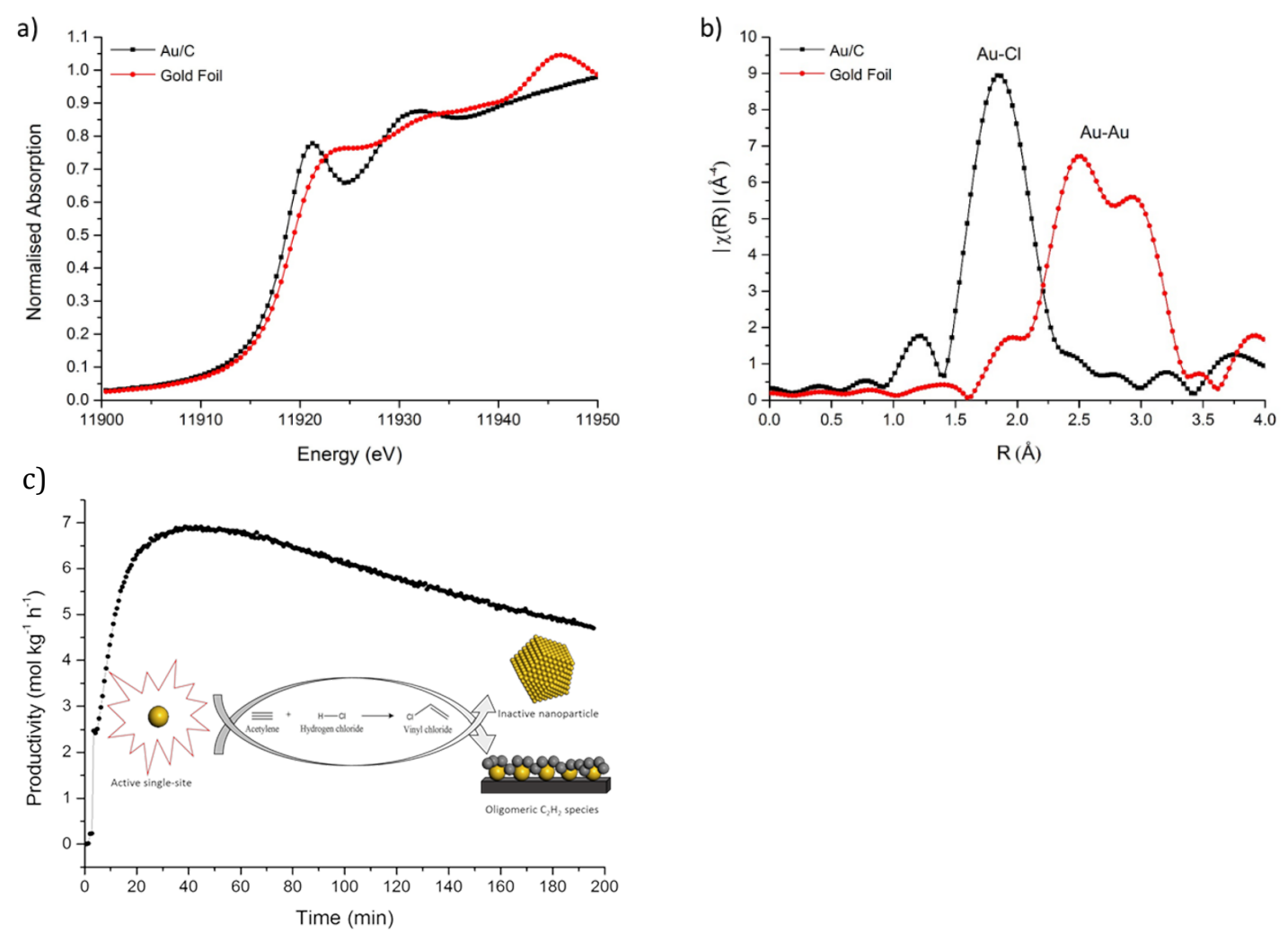

Figure 2. Au $\mathrm{L}_{3}$-edge a) normalised XANES and b) Fourier transform of $\mathrm{k}^{3}$-weighted $\chi$ EXAFS spectra of the $\mathrm{Au} / \mathrm{C}$ catalyst (black squares) and a gold foil reference material (red circle), c) Schematic representation of the deactivation pathways of single-site $\mathrm{Au} / \mathrm{C}$ catalyst during the acetylene hydrochlorination reaction

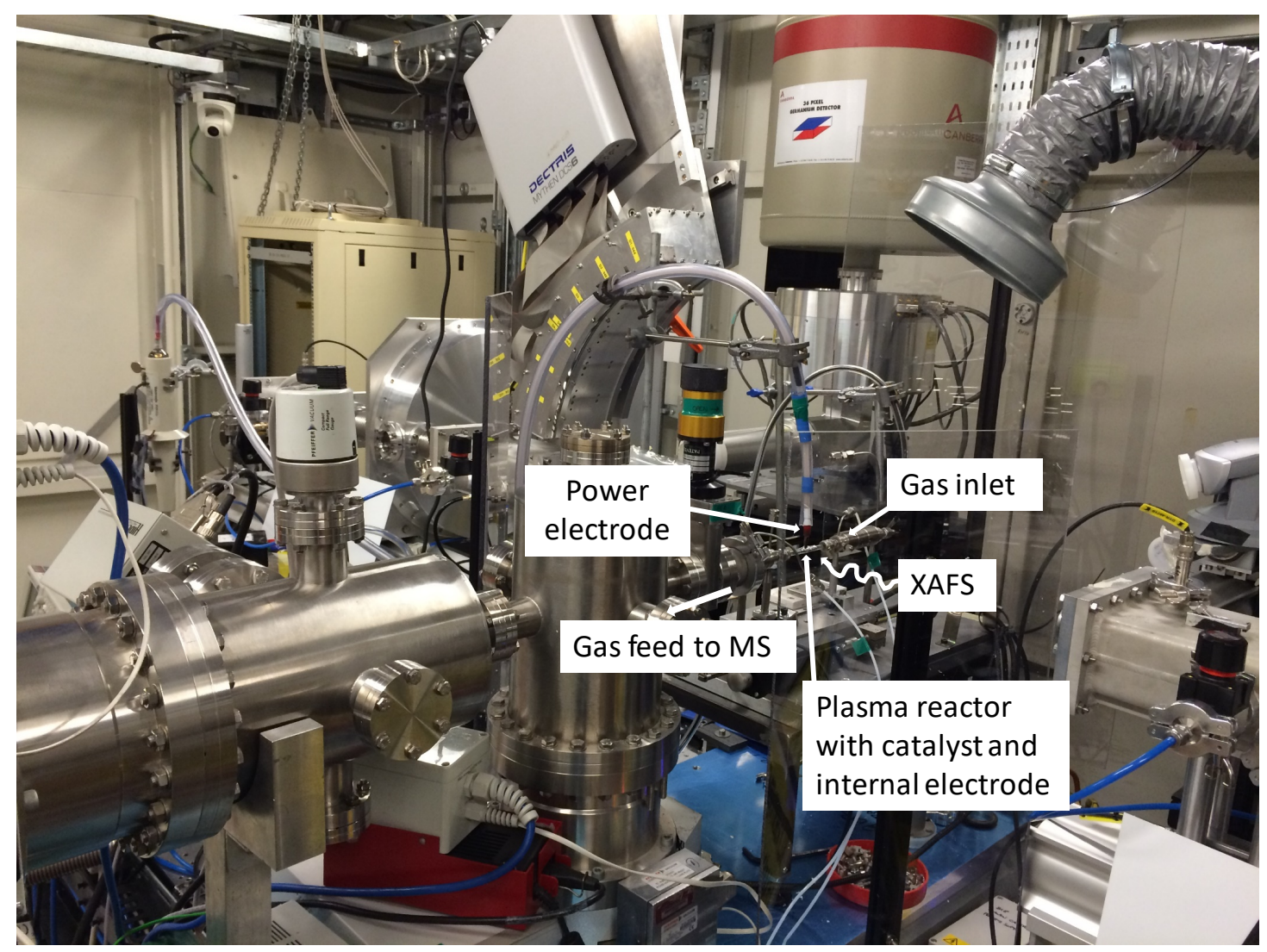


Figure 3. Setup for plasma-assisted gas phase measurements and XAFS spectroscopy. 